\title{
JERARQUÍA Y SISTEMA DE CIUDADES EN MÉXICO
}

\author{
Luis UNIKEL \\ $\mathrm{y}$ \\ ANDRÉS NECOCHEA* \\ El Colegio de México
}

I. IN TRODUCCIÓN

E N ESTE TRABAJo se presentan los primeros resultados de un estudio sobre jerarquía y sistema de las 38 ciudades que en 1960 contaban con 50000 y más habitantes.

Este ensayo forma parte del análisis descriptivo del proceso de urbanización en México que se está llevando a cabo en el Centro de Estudios Económicos y Demográficos de El Colegio de México.

El trabajo se ha dividido en tres partes. La primera analiza a los centros urbanos por sus características de "lugar centrar', es decir, como centro productor y distribuidor de bienes y servicios para la población residente y la de su zona de influencia." Para el efecto se construyó un índice de "alto grado de urbanismo" para cada centro." En una segunda parte se ha establecido un índice de "nivel de vida" de los habitantes de los centros urbanos en términos sociales, educativos y de vivienda. Por último, se ha determinado la posición que

* Una versión de este trabajo fue presentada a la Conferencia Regional Latinoamericana de Población, México, agosto 17-22, 1970. Se agradece la colaboración de Víctor Manuel Aquino en la recopilación de la información, y de Rosa Ma. R. de González y del Dr. Tomás Garza en la programación y asesoría estadística.

La elección de este universo de estudio se hizo de acuerdo al elevado peso relativo que tienen estas 38 ciudades en el total de la población urbana: localidades con 15000 y más habitantes, $83 \%$ en 1960.

Véase Luis Unikel, et al., El proceso de urbanización en México, anteproyecto de investigación, El Colegio de México (mimeografiado), abril de 1967.

3 Se ha utilizado el concepto de "lugar central" de Christaller y modificado por Losch y otros. Estos autores han trabajado sólo con el sector "servicios". En el presente trabajo se ha adicionado la variable valor agregado industrial. Un buen estudio sobre la materia se encuentra en Osear Yujnovsky y Carlos R. Tobar, "Esquema de asentamientos urbanos en un área de la mesopotamia argentina" en el Simposio de Geografía Urbana, editado por la Comisión de Geografía del Instituto Panamericano de Geografía e Historia, Río de Janeiro, 1968. Una elaboración más completa se tiene en Marie-Andrée Prost, La hiérarchie des villes en fonction de leurs activités de commerce et de service, París, Gauthier-Villars, 1965. )

4 Los elementos que definen la importancia relativa de una ciudad de acuerdo al concepto de "lugar central" son aquellas actividades de alto grado de especialización. El índice de "alto grado de urbanismo", de acuerdo con este criterio, comprende variables representativas de las funciones que, generalmente, desempeñan las ciudades importantes. 
cada centro ocupa dentro del campo de fuerzas del "espacio económico"s del sistema urbano, mediante el análisis de los flujos de vehículos, pasajeros y toneladas de carga industrial entre pares de centros. Este análisis se ha hecho mediante modelos gravitacionales y del potencial gravitacional.

Debido a limitaciones de espacio la presente exposición se concentra en los aspectos metodológicos del trabajo; se presentan además, en líneas generales, algunos resultados.

I I. ALTO GRADO DE URBANISMO

Para entender las funciones que desempeña una ciudad es necesario considerar un contexto más amplio que el de sus límites físicos, puesto que en la generalidad de los casos, éstas satisfacen necesidades de la propia población residente, como también de la que vive en su i periferia y que en algunos casos alcanza a todo el país. Por lo tanto, establecer una jerarquía entre los centros urbanos implica tomar en cuenta estos tres niveles geográficos — ciudad, su periferia y el paíspara poder determinar la importancia relativa de la ciudad dentro del sistema en su conjunto.

Tradicionalmente se ha tratado de establecer jerarquías de ciudades considerando las actividades de comercio y los servicios especializados que ésta presta a su periferia inmediata tanto como a sí misma.' Esta forma de análisis parte del modelo teórico presentado por Christaller en su difundida teoría de "lugares centrales". Tal teoría no considera el que centros urbanos de tamaño mediano puedan tener funciones industríales o de servicio altamente especializado, tales como la petroquímica, la siderúrgica y algunas actividades financieras, las cuales pueden abarcar mercados de alcance nacional e internacional.

El establecimiento de una jerarquía entre las 38 principales ciudades de México se ha hecho mediante el uso de un grupo de variables, medidas en términos absolutos, bajo el supuesto de que a mayor índice de "alto grado de urbanismo", mayor es la importancia relativa de la ciudad en el conjunto urbano del país. (Véase el cuadro 1.)

/ Para establecer la jerarquía se consideró necesario obtener un

5 Según Boudeville el espacio económico es una de las dimensiones del espacio geográfico que utiliza la serie de abstracciones de las cuales se vale el espacio matemático para representar hechos y resultados. No es un espacio real, es una abstracción pura... útil sobre todo en los análisis de actividades localizadas en un espacio geográfico. Este concepto permite entender mejor las relaciones entre centros de concentración de población y de actividades - regiones, metrópolis- en términos de interacción entre ciudades y del centro con su periferia, para llegar a los conceptos prácticos de jerarquía urbana y regiones de planificación. Véase Jacques-Raoul Boudeville, "La notion d'espace" en Boudeville, compilador, L'espace et les pôles de croissance, Paris, Presses Universitaires de France, 1968.

$f$ «Para mayores detalles sobre los conceptos de modelos gravitacionales y po-

$\mathrm{x}$ tencial gravitacional véase Walter Isard et al., Methods of Régional Analysis,

I Cambridge, MIT Press, 1960.

7 Un buen ejemplo de lo que se trata de explicar aquí se encuentra en Marie-Andrée Prost, op. cit. 
Cuadro 1

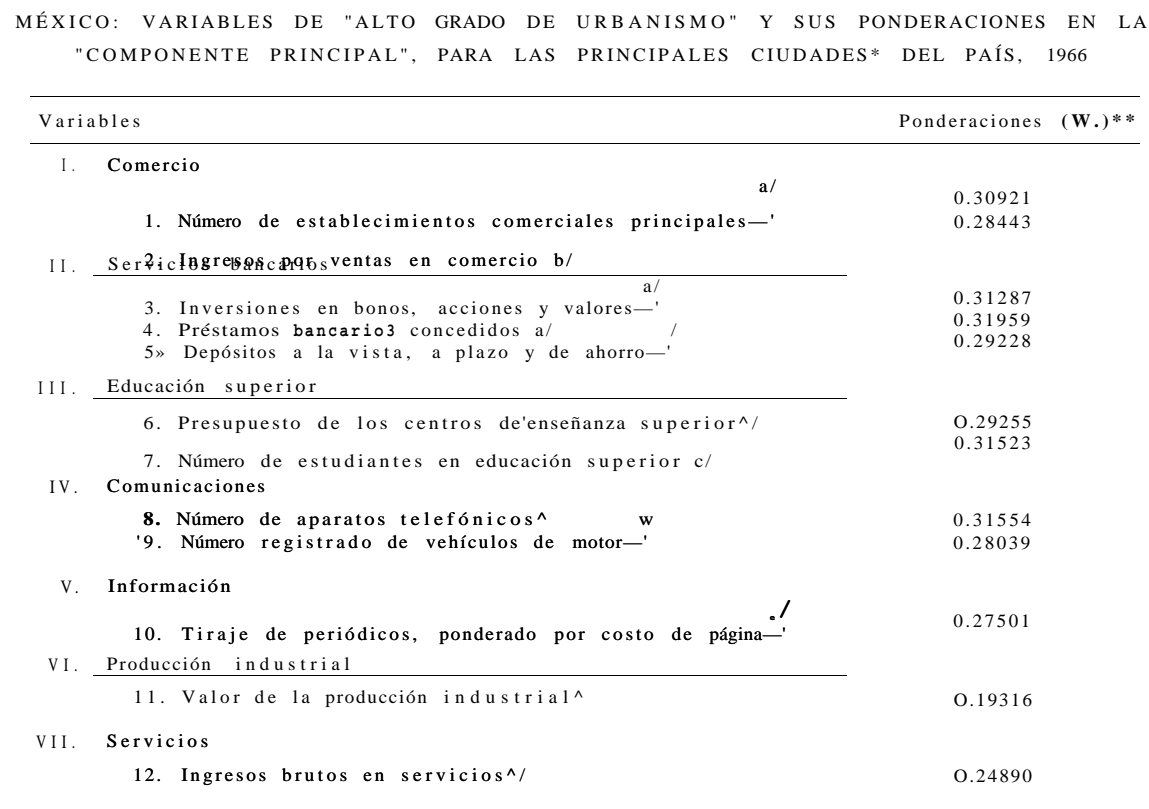

Fuentes: "Marynka Olizar, Guía a los mercados de México, 1968 ; \& Censo General de Comercio, Servicios e Industria, 1965, SIC (datos no publicados); o Dirección General de Enseñanza Superior e Investigación Científica, Departamento de Estadística Escolar, Secretaría de Educación Pública, 1966 (datos no publicados); $d$ Secretaría de Comunicaciones y Transportes Dirección General de Telecomunicaciones, 1966 (datos no publicados); e Medios Publicitarios Mexicanos, Departamento Editorial, 1966.

* Los datos utilizados en este análisis corresponden a la "ciudad central", con excepción de la ciudad de México y de Tampico, en las cuales la información abarca al Distrito Federal y a Tampico con Ciudad Madero, respectivamente.

** Las ponderaciones se obtuvieron con 37 ciudades (observaciones). Se excluyó a la ciudad de México debido al peso excesivo que ésta tiene en el cálculo de los coeficientes de correlación.

índice único y particular para cada centro, que tuviera las condiciones de ser comparable con los índices de las demás ciudades. Para ello, se utilizó el método de las "componentes principales" que es un caso particular del análisis factorial. ${ }^{8}$ El índice de "alto grado de urbanismo" se obtuvo de la primera componente que tiene la particularidad de que maximiza el porciento de varianza explicada por el conjunto de las 38 observaciones (ciudades) de las 12 variables utilizadas (véase el cuadro 1). La forma práctica de operar tal método es, una vez conocidos los factores de ponderación $\left(\mathrm{W}_{i}\right)$ de cada variable, aplicárselos

8 En los cálculos se utilizó un programa de biblioteca diseñado por Charles L. Clark, Factor Analysis Program for the Burroughs Computer, Detroit, Michigan, diciembre de 1964, Para una exposición completa de lo que es el análisis factorial, véase H. H. Harman, Modem Factor Analysis, University of Chicago Press, 1960. 
a la misma variable estandarizada' y luego sumar los resultados de esta operación para todas las variables de cada centro. El resultado de la suma es el "índice" (véase el cuadro 1). Éste constituye una síntesis altamente representativa del fenómeno estudiado, ya que explica el $72.9 \%$ de la varianza total."

I I . NIVEL DE VIDA

El índice de "nivel de vida" se calculó para determinar qué tamaño de ciudades presentan condiciones más favorables en cuanto al ^ bienestar general de su población. Mediante este índice se pretende verificar, como primera aproximación, si a un mayor tamaño de población corresponde un nivel de vida menor, que es la hipótesis comúnmente formulada.

Lo anterior es de especial interés para el caso de México, por lo mucho que se ha especulado en relación con los efectos negativos de la elevada concentración de la población y las actividades económicas en unas cuantas ciudades, particularmente en la de México.

En vista de que no se dispuso de información actualizada, los datos utilizados para el cálculo del índice fueron los censales de 1960. El estudio sobre nivel de vida se ha concentrado particularmente en variables educativas, de vivienda, vestuario y mobiliario del hogar.

Al igual que en la primera parte del estudio, se consideró conveniente contar con un índice único de nivel de vida para cada ciudad. Por esta razón, se aplicó el mismo método de análisis de la componente principal, expresando en este caso todas las variables en términos porcentuales con respecto al tamaño de la población. También se determinaron los pesos (W.i) en la misma forma antes expuesta (véase el cuadro 2).

El porciento de varianza explicada por la componente principal fue de $56.5 \%$, lo que si bien es relativamente bajo, puede considerarse aceptable. Por ello, el análisis de un mayor número de componentes parece recomendable para explicar un mayor porciento de la varianza total.

\section{INTEGRACIÓN DEL SISTEMA URBANO}

Hasta ahora se han analizado las ciudades del sistema en función de algunas de sus características internas. Si aceptamos la defini-

9 Dicha variable tiene media cero y varianza igual a uno y se obtiene aplicando

la fórmula siguiente: $X_{n}=\frac{x-x}{s}$ en que $x$ es una observación de la variable, $x$ es la media de las 38 observaciones del grupo de ciudades y 5 es la desviación estándar.

i'O En vista de la elevada correlación entre las variables analizadas, se juzga necesario llevar a cabo en un trabajo posterior una selección de aquellas más representativas del fenómeno.

n Debido a la poca disponibilidad de información, sólo se han utilizado algunas variables de nivel de vida sin la pretensión de que cubran toda la dimensión del concepto. 


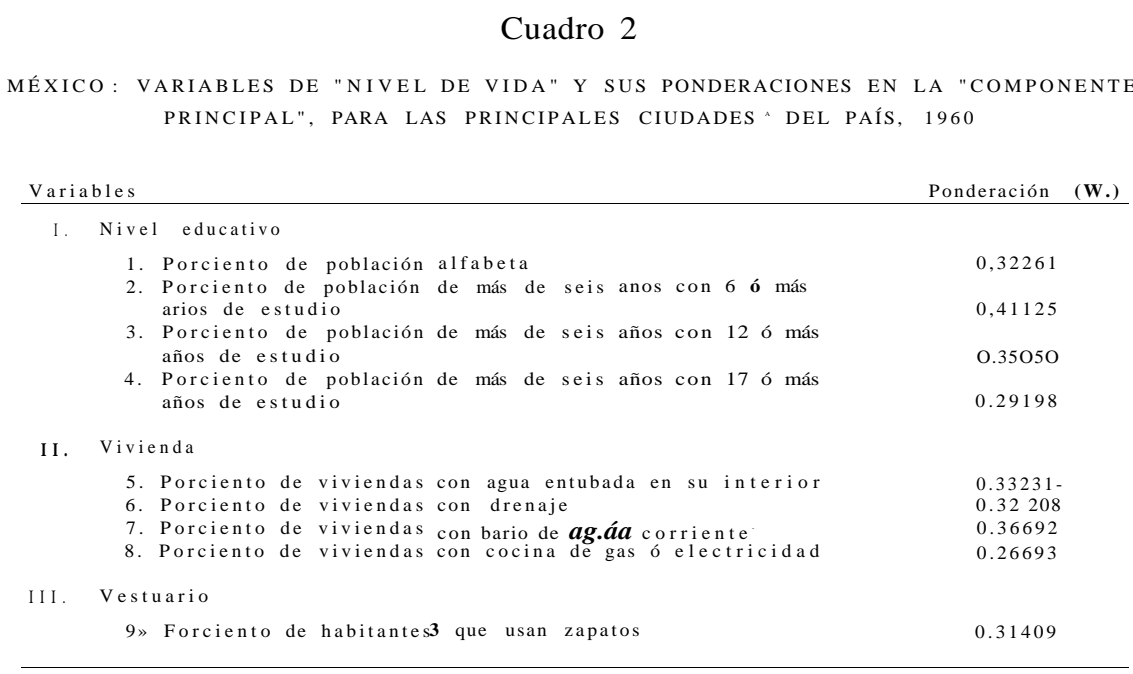

Fuente: Censo general de población, SIC, 1960.

a Los datos utilizados en este análisis corresponden a los municipios que contienen a las ciudades centrales, cuya población abarca, en 23 casos, más del $75 \%$ de la población municipal, y en 14 casos, entre el $49 \%$ y el $75 \%$ (Culiacán presentó una relación de $41 \%$ ). Debido a que las variables, vivienda, vestuario y la mayoría de las de nivel educativo, corresponden a características de vida predominantemente urbana, se considera que el dato municipal, en términos relativos, es altamente representativo de las ciudades estudiadas, aun en los casos en que la proporción de población ciudad a municipio sea inferior al $75 \%$. Una prueba indirecta de ello se tiene en que la PEA no agrícola en estos últimos casos fue en 1960, superior, en promedio, al $80 \%$ (en Culiacán fue del $56 \%$ ). En el caso de la ciudad de México, se consideró al Distrito Federal.

ción de sistema como "un conjunto de elementos en interacción", quedan por determinar las interrelaciones entre las ciudades.

Para ello, se han seleccionado los modelos gravitacionales como instrumento básico de trabajo ya que se cuenta con cinco matrices de flujo interurbano de vehículos, personas y toneladas de carga industrial. ${ }^{13}$ Las observaciones corresponden a estudios de origen y destino en México realizadas entre 1963 y 1968, cuya media ponderada se sitúa cerca de 1966.

El tratar las consideraciones teóricas sobre los modelos gravitacionales está fuera de los alcances de este trabajo; existe una amplia bibliografía sobre la materia. ${ }^{14}$ Para los fines de este estudio se ha

12 Ludwig von Bertalanffy, "General Systems Theory", General Systems, Núm. 1, 1956, p. 3.

La fuente de tales matrices son los estudios que la Oficina de Planeación y Programa de la Secretaría de Obras Públicas ha venido realizando a partir de 1963. La metodología de encuesta no ha sufrido alteraciones por lo que los datos son totalmente comparables y promediables entre sí. En el presente trabajo se utilizaron los 78 estudios disponibles. Con éstos se alcanza a cubrir casi la totalidad del país, quedando algunos espacios muy pequeños sin cubrir que carecen de importancia para la determinación del comportamiento del sistema urbano.

Para una buena bibliografía anotada respecto a modelos gravitacionales, consultar Gunnar Glasson, Distance and Human Interaction, a Review and Bibliography, Regional Science Research Institute, Filadelfia, 1965. También se puede 
utilizado la fórmula gravitacional general siguiente:

$$
F i,=-\underset{i j}{-} K
$$

en que, $\mathrm{F}_{4 i}$ es el flujo del centro $i$ al $/, \boldsymbol{M}_{i,} \boldsymbol{M}_{\boldsymbol{i}}$ son "masas gravitacionales" tales como la población, el valor de la producción, etc.; $d_{i f}$ es la mejor distancia que media entre el centro $i$ y el / por carretera; ${ }^{15}$ $\boldsymbol{K}$ es una constante que depende de la magnitud de los valores no paramétricos; y $a, b$ y $c$ son parámetros determinables por el comportamiento del sistema.

Mediante regresiones múltiples se estimaron los parámetros utilizando diferentes "masas gravitacionales" — población, toneladas de carga industrial, etc. - de acuerdo con la índole del flujo. Las matrices que se analizaron fueron de dos tipos: las dos primeras, de flujo total entre pares de centros urbanos; las tres restantes fueron de origen y destino entre pares de centros - matrices de vehículos, pasajeros y toneladas de carga industrial. En estas tres últimas matrices se analizó la parte superior en forma independiente de la inferior, ordenando las ciudades por tamaño decreciente de población. ${ }^{16}$ Esto se hizo para tener una estimación más exacta del comportamiento de los flujos: la parte superior es el flujo de $i$ hacia / y la inferior el opuesto. En esta forma se llega a tener dos grupos de parámetros para cada matriz de origen y destino - cada una consta de 612 observaciones-: uno aplicable al tránsito del centro mayor $i$ al menor ; y el otro a la relación inversa, del centro / al $i$.

Como "masas gravitacionales" se utilizaron la población total del área urbana de la ciudad $(P)$, la proporción de ventas en comercio al mayoreo respecto al total de ventas (C), el valor agregado industrial en 1960 (F) y el valor total de la producción industrial en 1965 ( T). Con el uso de combinaciones diferentes de estas variables en las regresiones se llegó a determinar un grupo de ecuaciones gravitacionales compuestas que explican en forma más satisfactoria el flujo interurbano.

A efecto de medir el grado de integración de cada ciudad al sistema en su conjunto, se relacionó el flujo real observado con el estimado. Este último se calculó mediante las ecuaciones gravitacionales previamente determinadas, obteniéndose cinco matrices de flujo y de origen y destino equivalentes con las observadas. El grado de integración al sistema urbano es un indicador del aprovechamiento de las ventajas competitivas de la localización de un centro $i$, con respecto a todos los demás. Un alto valor de este índice puede señalar dos situaciones para una ciudad: por una parte, que ésta pertenezca a un subsistema

ver Brian Berry y Alien Pred, Central Place Studies, Filadelfia, Regional Science Research Institute, 1965.

15 El uso del tiempo promedio de recorrido entre pares de ciudades es una medida más exacta que la distancia. Sin embargo, se optó por esta última, debido a la dificultad que significaba obtener el tiempo de recorrido.

$16 \mathrm{La}$ parte superior de las matrices corresponde a los elementos a la derecha de la diagonal y la inferior a los de la izquierda. 
con una alta interacción entre las ciudades que lo componen, o, que está altamente integrado al sistema nacional en su conjunto. La confrontación de los índices con el mapa de aforo de tránsito por carretera (en elaboración) permitirá saber si se trata de la primera o la segunda situación, o si es una ciudad aislada. Por último, se determinó el potencial gravitacional ${ }^{17}$ de cada centro urbano, entendido como la capacidad de generar flujo de vehículos, personas y bienes de consumo, desde otras ciudades en razón de la estructura de las actividades de la ciudad. Para el caso de México se estimaron los potenciales gravitacionales de población, turismo, industria y comercio. ${ }^{18}$ La determinación de tal pptencial se hizo mediante la fórmula: ${ }^{19}$

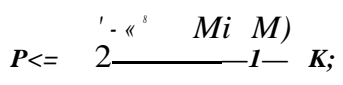

en que = potencial gravitacional del centro $i ; M_{,}$y $M_{i}$ son "masas gravitacionales" tales como la población, el valor de la producción, etc.; es la menor distancia que media entre el centro $i$ y el / por carretera; $\boldsymbol{K}$ es una constante que depende de la magnitud de los valores no paramétricos y $a, b$ y $c$ son parámetros determinables por el comportamiento del sistema.

En vista de que se obtuvieron cuatro valores de potencial gravitacional para cada ciudad, resultado de la aplicación de diferentes masas gravitacionales - población, camas de hotel, producción industrial e importe de las ventas en comercio- y de que era conveniente contar con un solo índice de potencial, se aplicó el método de las "componentes principales" para determinarlo. ${ }^{20}$

V. EXPOSICIÓN DE ALGUNOS RESULTADOS

Está fuera de los propósitos de este trabajo hacer un análisis exhaustivo de los resultados del trabajo, por lo que a continuación se presenta una síntesis resumida de los mismos en los cuadros 3, 4 y 5 .

Un primer resultado interesante es la correlación de rangos positiva y alta $(r=0.84)$ existente entre el tamaño de ciudad y el índice de "alto grado de urbanismo". Es decir, que a medida que aumenta

Dirección de Planeación y Programa, S.O.P. Estudios de Origen y Destino. Los datos utilizados para cada uno de ellos fueron: población proyectada a 1966, número de camas de hotel, valor de la producción industrial en 1965 e importe total de las ventas en comercio en 1965 en cada ciudad.

Esta fórmula considera al centro urbano como una parte de su propio potencial gravitacional. Por ello al denominador se le ha sumado 1; en caso contrario el valor de la expresión sería indefinido en la observación del centro consigo mismo por ser la distancia igual a cero.

Existe un estudio bastante completo sobre el potencial gravitacional en México en que se llegó a determinar las curvas equipotenciales. Ver Robert Tata, "El'uso de los análisis sobre el potencial de espacio localizado para el estudio de la industrialización mexicana", en Conferencia Regional Latinoamericana de la Unión Geográfica Internacional, editado por la Sociedad Mexicana de Geografía y Estadística, México, D. F., 1966, tomo n, pp. 625. 
Cuadro 3

MÉXICO: ÍNDICES COMPUESTOS DE JERARQuía Y Sistema DE CIUdAdes, 1966a

\begin{tabular}{|c|c|c|c|c|c|c|c|c|c|c|}
\hline \multirow{3}{*}{$\begin{array}{c}\text { Ciudades por orden } \\
\text { decreciente de ta- } \\
\text { en } 1966 \mathrm{~d} /\end{array}$} & \multirow{2}{*}{\multicolumn{2}{|c|}{$\begin{array}{c}\text { Alto grado de } \\
\text { urbanismo }\end{array}$}} & \multirow{2}{*}{\multicolumn{2}{|c|}{$\begin{array}{l}\text { Nivel de } \\
\text { vida }\end{array}$}} & \multirow{3}{*}{$\begin{array}{l}\text { Potencial } \\
\text { oional }\end{array}$} & \multicolumn{5}{|c|}{ Grado de integración ais } \\
\hline & & & & & & \multicolumn{3}{|c|}{ Sistema urbano } & \multicolumn{2}{|c|}{$\begin{array}{c}\text { Hesto del sistema } \\
\text { urbano } \mathbf{S i} \\
\end{array}$} \\
\hline & índice^ & Rango^/ & Indioe & Rango & & ind & lice & Rango & Indioe & Rango \\
\hline Ciudad de México & 20.229 & 1 & 3.697 & 2 & 11680 & si & & & $s i$ & \\
\hline Guadalajara & .950 & 3 & 2.840 & 5 & $-\quad 34$ & - & 979 & 27 & -1212 & 29 \\
\hline Monterrey & 1.780 & 2 & 4.090 & 1 & 281 & -1 & 256 & 7 & 1.003 & 11 \\
\hline Ciudad Juárez & -.544 & 10 & 0.734 & 17 & -.339 & & 445 & 14 & 1475 & 6 \\
\hline Puebla & -.074 & 5 & 2.700 & 6 & -370 & & 645 & 11 & -1336 & 30 \\
\hline Torreen & -.576 & 11 & 1.358 & 12 & -369 & & 583 & 13 & 651 & 15 \\
\hline Mexioali & .648 & 4 & -1.925 & 29 & -1368 & & 632 & 12 & 652 & 14 \\
\hline León & -.635 & 16 & -1.609 & 28 & -375 & - & 758 & 25 & 403 & 23 \\
\hline Tijuana & -.400 & 7 & 1.246 & 15 & -323 & 1 & 476 & 6 & 1386 & 9 \\
\hline Chihuahua & -.341 & 6 & 2.318 & 7 & -373 & & 704 & 10 & $\bullet 1438$ & 7 \\
\hline Tampico & -.541 & 9 & 2.984 & 3 & -368 & - & 534 & 23 & 87 & 17 \\
\hline San Luis Potosí & -.616 & 15 & 0.224 & 20 & -375 & -1 & 500 & 32 & 132 & 20 \\
\hline Mérida & -.589 & 13 & 0.595 & 19 & -376 & -2 & 062 & 33 & - 2776 & 35 \\
\hline Veracruz & -.530 & 8 & 2.869 & 4 & -259 & & 847 & 9 & 837 & 13 \\
\hline Hermosillo & -.581 & 12 & 1.287 & 14 & -385 & & 126 & 18 & 26 & 19 \\
\hline Aguascalientes & -.745 & 21 & 1.299 & 13 & -387 & - & 568 & 24 & 326 & 21 \\
\hline Morelia & -.702 & 19 & - 1.117 & 23 & -388 & -2 & 148 & 35 & -1700 & 31 \\
\hline Matamoros & -.827 & 30 & -0.807 & 22 & - 339 & -1 & 450 & 31 & 751 & 26 \\
\hline Durango & -.788 & 26 & -1.560 & 27 & -392 & - & 145 & 21 & 502 & 16 \\
\hline Orizaba & -.871 & 35 & 1.731 & 10 & - 393 & -1 & 180 & 29 & 1340 & 10 \\
\hline Nuevo Laredo & -.776 & 25 & 2.264 & 8 & -386 & & 691 & 4 & 2529 & 2 \\
\hline
\end{tabular}




\begin{tabular}{|c|c|c|c|c|c|c|c|c|c|c|c|}
\hline Saltillo & - .786 & 18 & 0.860 & 16 & -369 & 3 & 797 & 2 & 4 & 575 & 1 \\
\hline Culiacán & -.652 & 17 & -3.563 & 36 & -392 & 1 & 700 & 5 & 2 & 116 & 5 \\
\hline Reynosa & -.863 & 34 & -2.568 & 33 & -392 & -1 & 173 & 28 & & 10 & 18 \\
\hline Toluoa & $=\cdot 611$ & 14 & -2.426 & 32 & -390 & 3 & 270 & 3 & -2 & 698 & 34 \\
\hline Irapuato & $=.842$ & 32 & -2.327 & 31 & -392 & - & 375 & 22 & & 867 & 12 \\
\hline Mazatlán & - 769 & 24 & -1.243 & 25 & -340 & 1 & 079 & 8 & 2 & 287 & 4 \\
\hline Ciudad Obregón & -.719 & 20 & -1.226 & 24 & -387 & - & 904 & 26 & & 663 & 25 \\
\hline Oaxaca & $=.828$ & 31 & -1.405 & 26 & -386 & -2 & 068 & 34 & $=2$ & 168 & 33 \\
\hline Caernavaca & -.798 & 27 & 0.659 & 18 & -371 & & $£ /$ & - & -2 & 826 & .36 \\
\hline Tepio & $-\cdot 363$ & 33 & - $\quad 1.975$ & 30 & -392 & & 405 & 15 & 1 & 394 & 8 \\
\hline Querétaro & .826 & 29 & -2.680 & 34 & -391 & & 252 & 17 & -1 & 058 & 28 \\
\hline Celaya & $\bar{z} \cdot 884$ & 37 & -3.090 & 35 & -393 & & 296 & 16 & 2 & 472 & 3 \\
\hline Jalapa & - $\cdot 756$ & 23 & 1.740 & 9 & - 394 & -2 & 223 & 36 & -2 & 126 & 32 \\
\hline Villahermosa & - $\bullet 879$ & 36 & -3.972 & 38 & - 388 & - & 84 & 20 & & 805 & 27 \\
\hline Pachaca & -.824 & 23 & -1.702 & 11 & -395 & & 25 & 19 & -3 & 441 & 37 \\
\hline Aoapulco & - $\cdot 747$ & 22 & -3.749 & 37 & 874 & 4 & 369 & 1 & - & 612 & 24 \\
\hline Ciudad Victoria & $\begin{array}{r}\cdot 912 \\
-\end{array}$ & 38 & 0.041 & 21 & -394 & -1 & 396 & 30 & - & 376 & 22 \\
\hline
\end{tabular}

a Estos índices son el resumen de una serie de índices parciales que se publicarán por el Centro de Estudios Económicos y Demográficos, en un artículo más amplio sobre el tema,

b Este índice corresponde a 1960. Para mayores detalles véase la nota a del cuadro 2. Esta nota corresponde al índice de nivel de vida de la columna siguiente. [Ed.]

c Estos cálculos corresponden a las ciudades centrales excepto en los casos de la ciudad de México y Tampico. Véase la nota del cuadro 1.

a La proyección de la población de las "áreas urbanas" se hizo con base en la tasa de crecimiento geométrico observada en el período 1950-1960. Esta proyección se está actualizando con el dato censal de 1970 y mediante un método más refinado,

e Por resto del sistema urbano se entiende a todas las ciudades excluyendo solamente a la ciudad de México.

'No se dispuso de ningún estudio de origen y destino entre ciudad de México y Cuernavaca.

g El índice obtenido para la ciudad de México se excluyó por considerarse que su valor, demasiado bajo, no refleja la realidad. Esto se debe a que la función, determinada mediante la regresión, está influida por el peso que tienen en ella el elevado número de ciudades de tamaño medio. 
el tamaño de la población de la ciudad, es mayor su especialización funcional. Esto implica que las ciudades de mayor población tienden a concentrar un mayor número de actividades especializadas, de importancia regional y nacional, tanto económicas, culturales y financieras.

Por otra parte, el tamaño de población y el índice "nivel de vida" están asociados positivamente, aunque en forma débil: la correlación de rangos fue de 0.57. Se observa, al dividir el conjunto de ciudades en tres grupos, que en el que contiene a las 13 ciudades de mayor población se encuentran los más altos índices de nivel de vida; en cambio, el grupo de las ciudades menores presenta sólo tres casos con niveles de vida con valores medianos, siendo bastante bajos los valores restantes (véase el cuadro 3 ).

Sin embargo, el comportamiento estadístico de las variables antes

\section{Cuadro 4}

MÉXICO: ÍNDICE DE PRIMACÍA DE LA POBLACIÓN Y DE VARIOS ELEMENTOS DE "ALTO GRADO DE URBANISMO", 1966

\begin{tabular}{|c|c|c|c|c|c|}
\hline \multirow{2}{*}{ Conceptos } & \multicolumn{5}{|c|}{ índice de primaoía de»-' } \\
\hline & 2 ciudades & 4 ciudades & 6 ciudades & 8 ciudades & 10 ciudades \\
\hline Población, 1966 b/ & 5.51 & 2.51 & 1.99 & Í. 68 & 1.50 \\
\hline $\begin{array}{l}\text { índice de "alto erado de } \\
\text { urbanismo" c/ }\end{array}$ & 11.77 & 6.53 & 5.94 & 5.62 & 5.47 \\
\hline $\begin{array}{l}\text { N\&mero de establecimien- } \\
\text { tos comerciales princi } \\
\text { pales }\end{array}$ & $4-78$ & 2.08 & 1.56 & Í.31 & 1.14 \\
\hline $\begin{array}{l}\text { Ingresos por ventas en } \\
\text { comercio }\end{array}$ & 9.33 & 3,97 & 3.03 & 2.51 & 2.21 \\
\hline Servicios bancarios- & 6.96 & 3.87 & 3.22 & 2.83 & 2.55 \\
\hline $\begin{array}{l}\text { Presupuesto de los cen- } \\
\text { tros de enseñanza su- } \\
\text { perior }\end{array}$ & 8,04 & 4.47 & 3.51 & 3.02 & 2.73 \\
\hline $\begin{array}{l}\text { Numero de estudiantes en } \\
\text { educación superior }\end{array}$ & 6.71 & 2.75 & 2.36 & 2.07 & 1.85 \\
\hline $\begin{array}{l}\text { Número de aparatos tele- } \\
\text { fónicos }\end{array}$ & 10.73 & 4.47 & 3.66 & 3.12 & 2.78 \\
\hline $\begin{array}{l}\text { Número registrado de ve- } \\
\text { hículos de motor }\end{array}$ & 6.96 & 2.52 & 1.90 & 1.65 & 1.49 \\
\hline $\begin{array}{l}\text { Tiraje de periódicos, pon } \\
\text { derado por co3to de pá } \\
\text { gina }\end{array}$ & 13.21 & 6.78 & 4.99 & 4.11 & 3.57 \\
\hline $\begin{array}{l}\text { Valor de la producción in } \\
\text { dustrial }\end{array}$ & 5.08 & 3.00 & 2.51 & 2.22 & 2.02 \\
\hline $\begin{array}{l}\text { Ingresos brutos en servi- } \\
\text { cios }\end{array}$ & 10.91 & 4.97 & 3.71 & 3.25 & 2.94 \\
\hline
\end{tabular}

\& Este índice se calculó mediante la expresión: $I_{n}(n)=\frac{}{P_{n_{2}}+P_{\mathrm{c}_{2}}+\mathrm{F}_{4}+\ldots+P_{n}}$ en donde $P_{v} P_{2}, P_{s}, \mathrm{P}_{4}, \ldots P_{n}$ son los valores de las variables correspondientes a las ciudades que en esa fecha ocupaban los rangos $1,2,3,4$ y $n$, respectivamente.

k Población de áreas urbanas calculada para 1966.

c Véase cuadro 3.

d Incluye las variables: inversiones en bonos, acciones y valores; préstamos bancarios concedidos y depósitos bancarios (a la vista, a plazos y ahorro). 
analizadas no permite hacer inferencias definitivas sobre los aspectos estudiados del nivel de vida en las ciudades del país según el tamaño de su población.

La jerarquía de las 38 ciudades determinada mediante el índice de "alto grado de urbanismo" presenta como rasgo característico una primacía mayor que la de la población, ya de por sí considerada elevada (véase el cuadro 4). En otras palabras, la concentración de actividades especializadas es en todos los casos significativamente mayor que la de la población, alcanzando el máximo en la impresión de periódicos, casi tres veces superior.

Esto expresa con claridad el fuerte centralismo existente en México en población y, particularmente, en actividades económicas, culturales y sociales. De aquí se desprende la enorme dificultad que existe para que una política de descentralización de población sea exitosa, pues ello implicará activar contra fuerzas de concentración de actividades aún más poderosas que las de la población.

$\mathrm{Si}$ se trata de establecer grupos de ciudades de acuerdo con su posición dentro de la jerarquía se tiene en primer lugar, obviamente, a la ciudad de México, que concentra la mayor parte de las actividades analizadas, sin ninguna posibilidad de que alguna otra ciudad llegue a igualarla por un largo espacio de tiempo. Siguen, en un segundo grupo, Monterrey y Guadalajara, que podrían llamarse centros de importancia nacional; existe, eso sí, una gran diferencia entre ellas y es en el grado de desarrollo industrial, mucho mayor en Monterrey que en Guadalajara. En tercer grupo se clasifican Puebla, Chihuahua, Mexicali y Tijuana, como centros de grandes regiones. En un cuarto nivel se agrupan algunas ciudades fronterizas y especialmente las portuarias. Por último, se presenta un grupo bastante homogéneo de ciudades de mayor o menor importancia relativa dentro de sus regiones, que constituyen el resto del sistema urbano.

Por lo tanto, el sistema urbano de México — considerando los 38 principales centros - está formado por cuatro grupos de ciudades: el primero con una, el segundo con dos, el tercero con ocho y el cuarto con veintisiete ciudades. Esta distribución se aleja mucho de la ideal, definida por Christaller y analizada por Berry."

Las conclusiones de Berry se pueden trasladar al caso de México como situación típica de un país en desarrollo, ya que la población y en mayor grado las actividades económicas distan mucho de seguir la ley de Pareto en su distribución rango-tamaño.

En términos de integración al sistema urbano nacional, la ciudad de México destaca en cuanto que genera más de la mitad de los viajes totales que se hacen en el país. En otras palabras, por cada viaje de cualquiera de los tipos estudiados que se genere en una ciudad cualquiera con respecto a otra que no sea la capital, esta última genera más de un viaje con la ciudad en cuestión.

Los parámetros obtenidos en las regresiones para diversos tipos de flujos muestran varios hechos destacables. En general, en la generación de flujos en ambos sentidos, el peso de la ciudad mayor es más

,.; ${ }^{21}$ B. Berry/"Cities as Systems Within Systems of Cities", en J. Friedman y William Alonso, compiladores, Regional Development and Planning, Cambridge, The M I T Press, 1964, pp. 116-137. 
elevado que el de la menor. La "fricción del espacio", medida en este caso como la menor distancia que media por carretera entre un par de centros, es muy similar en ambos sentidos (parámetro $c$ de $i$ a ; y de ; a i). Además, es mayor para el transporte de productos industriales que en la movilidad de vehículos y pasajeros, de 1.71 a 1.63 contra 1.63 a 1.00 , respectivamente (cuadro 5).

\section{Cuadro 5}

MÉXICO: PARÁMETROS DETERMINADOS MEDIANTE LA ECUACIÓN GRAVITACIONAL SIMPLE, CON POBLACIÓN Y DISTANCIA, 1966 a

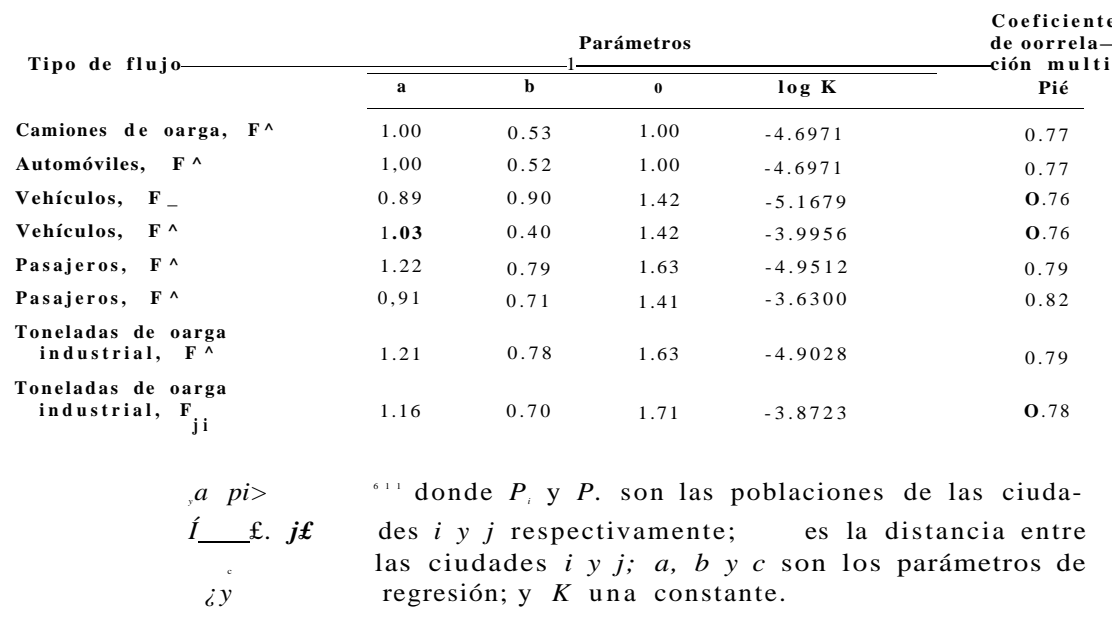

El sistema urbano en México parece tener una integración bastante grande, ya que los parámetros de la distancia (dy) son similares a los de algunos países desarrollados ${ }^{22}$ (véase el cuadro 5). Este comportamiento es estable en las ecuaciones compuestas - que utilizan combinaciones de "masas gravitacionales" y que no se presentan en este trabajo- en todos los factores previamente mencionados, especialmente en distancia. Otro hecho de interés es que hay varías ciudades que dependen de la ciudad de México, como se desprende al excluirla del análisis de la relación entre el flujo real y el estimado. Notables en este sentido son los casos de Puebla, Toluca, Querétaro, Pachuca y, muy probablemente, Cuernavaca, todas ciudades cercanas a la capital. Acapulco es un caso similar aunque a una distancia mucho mayor.

En el cuadro 3 se observa que estas ciudades tienen un grado de integración muy alto al sistema urbano y que, quitando a la ciudad de México, decae significativamente. Esto implica una relación muy débil con el resto de las ciudades del sistema estudiado. Otro hecho

22 Los parámetros del factor distancia son: + 1.67 y cerca de +2.0 , respectivamente, en Francia y los Estados Unidos. Véase Remigio Valdés G., "El flujo y la composición del tránsito carretero en México", Comunicaciones y Transportes México, D. F., abril-junio de 1966, núm. 2, p. 53. 
que cabe destacar es la conformación de un subsistema de ciudades en el del Bajío formado por Celaya, Irapuato y León (de los 38 centros urbanos estudiados) y seguramente, Guanajuato, Salamanca, Cortázar y otras ciudades menores no incluidas en el análisis. Se nota la existencia de otro subsistema con interrelaciones más débiles^el de las ciudades de la costa del Pacífico localizadas al norte de Guadalajara, con tendencia a gravitar sobre Mexicali y Tijuana, y, por lo tanto, probablemente, sobre los Estados Unidos de América. Orizaba y Jalapa tienden a su vez a gravitar sobre Veracruz, sin tener mayores relaciones con el resto del sistema ni entre sí. Por otro lado, destaca el aislamiento de ciudades como Mérida, Durango y Villahermosa.

Por último, de acuerdo con los cálculos del potencial gravitacional, los centros urbanos que sobresalen son la ciudad de México, Monterrey y Acapulco; la primera por su gran tamaño de población y actividad; Monterrey por su alta capacidad instalada en industria, y Acapulco por su importancia turística nacional e internacional. El resto de las ciudades del sistema conforman un grupo muy homogéneo y su importancia relativa tiene una correlación positiva con el número de sus habitantes. Esta homogeneidad con seguridad se reduce en forma significativa si se calcula el potencial gravitacional sin la ciudad de México. 